\title{
Extraction of polycyclic aromatic hydrocarbons from polyhydroxyalkanoates before gas chromatography/mass spectrometry analysis
}

\author{
Chiara Cavaliere $^{\mathrm{a}}$, Carmela Maria Montone ${ }^{\mathrm{a}}$, Anna Laura Capriotti ${ }^{\mathrm{a}, *}$, Giorgia La Barbera ${ }^{\mathrm{a}}$, \\ Susy Piovesana ${ }^{\mathrm{a}}$, Mauro Rotatori ${ }^{\mathrm{b}}$, Francesco Valentino ${ }^{\mathrm{a}}$, Aldo Laganà ${ }^{\mathrm{a}}$ \\ a Department of Chemistry, University of Rome "La Sapienza", Piazzale Aldo Moro 5, 00185 Rome, Italy

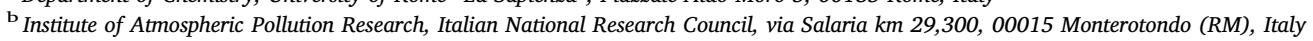

\section{A R T I C L E I N F O}

\section{Keywords:}

Biopolymers

Florisil clean-up

Gas chromatography/mass spectrometry

Isotopic dilution

Polycyclic aromatic hydrocarbons

Polyhydroxyalkanoates

\begin{abstract}
A B S T R A C T
Among the organic contaminants that could pass from waste to polyhydroxyalkanoates (PHAs), there are the polycyclic aromatic hydrocarbons (PAHs). For this reason, we have developed a rapid analytical method for the determination of sixteen PAHs in PHAs. PAHs were extracted by $n$-hexane, after matrix dispersion and crumbling into sand; the extract was purified by solid phase extraction using florisil as adsorbent. Recoveries in the range of 89-101\% were obtained for the deuterated analytes, except for the two with the lowest molecular weight. Trueness between $92 \%$ and $108 \%$ and within-laboratory precision (expressed as relative standard deviation) $\leq 18 \%$ were estimated for all the analytes. Gas chromatography/mass spectrometry was used for analyte determination. Method limits of quantification were suitable to assure that PAH presence in PHA biolpolymers is much below the limits set by European law for plastic materials. Indeed, analysis of two different PHA samples showed that contamination is limited to few compounds at non-concerning levels.
\end{abstract}

\section{Introduction}

Nowadays, in line with the Circular Economy approach, great effort is being devoted to reduce waste amount by reusing it and recovering high value substances. For example, it is possible to transform organic waste via anaerobic fermentation in volatile fatty acids (VFAs) [1], which can be used as a low cost external carbon source for the production of environmentally friendly products, such as polyhydroxyalkanoates (PHAs). PHAs are completely biodegradable and biocompatible polyesters with mechanical properties comparable to those of conventional oil based polymers [2]. The employment of organic fraction of municipal solid waste (OFMSW) as a new feedstock for microorganism-based PHA production is very attractive to reduce PHA high cost and increase their sustainability [3]. Nevertheless, it is necessary to guarantee suitable quality standards and the absence of incorporated chemical contaminants in the final product.

Polycyclic aromatic hydrocarbons (PAHs), constituted by two or more polycondensed aromatic rings, are mainly formed during the incomplete combustion of materials containing saturated hydrocarbons. $\mathrm{PAH}$ physicochemical properties (i.e., low vapor pressure and low solubility in water for high molecular weight PAHs, see Supplementary material Table S1), make them highly persistent and ubiquitous in the environment. PAHs can also reach the food chain, and their presence in the OFMSW has been reported [4-7].

The PAH adverse effects on human health are well known [8] and, accordingly to the International Agency for Research on Cancer (IARC) [9], benzo[a]pyrene is classified as carcinogenic to humans (group 1), whereas other PAHs as probably or possibly carcinogenic to humans

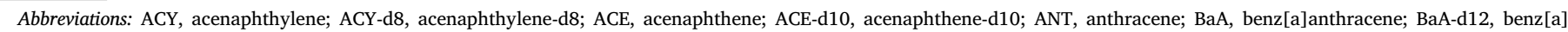

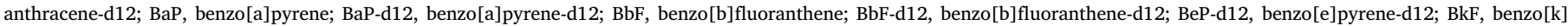

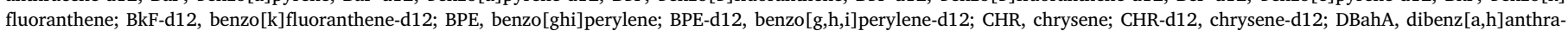

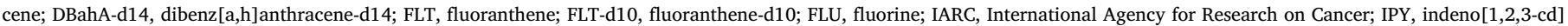

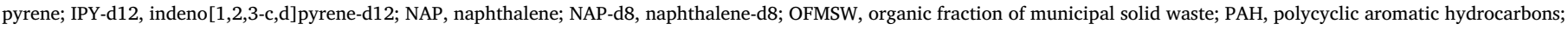

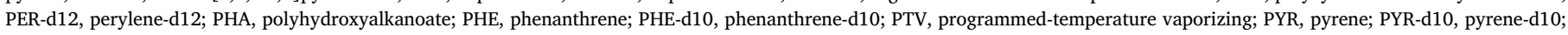
US EPA, United States Environment Protection Agency; VFAs, volatile fatty acids

* Corresponding author.

E-mail addresses: chiara.cavaliere@uniroma1.it (C. Cavaliere), carmelamaria.montone@uniroma1.it (C.M. Montone), annalaura.capriotti@uniroma1.it (A.L. Capriotti), giorgia.labarbera@uniroma1.it (G. La Barbera), susy.piovesana@uniroma1.it (S. Piovesana), rotatori@iia.cnr.it (M. Rotatori), francesco.valentino@uniroma1.it (F. Valentino), aldo.lagana@uniroma1.it (A. Laganà).
} 
(group 2A and 2B, respectively) (see Supplementary material Table S1).

The United States Environment Protection Agency (US EPA) has identified a list of sixteen PAHs as priority compounds for monitoring. Also, the European Union has issued directives and regulations aimed to reduce $\mathrm{PAH}$ presence into the environment $[10,11]$ and food [12]; in various articles (e.g. bicycles, household utensils, trolleys, clothing, footwear etc.) and toys, eight PAHs must have concentrations $<1$ and $0.5 \mathrm{mg} \mathrm{kg}^{-1}$ each, respectively (see Supplementary material Table S1) [11].

Several procedures are available for extraction of PAHs from various matrices $[13,14]$, whereas for their determination gas chromatographymass spectrometry (GC/MS) or high performance liquid chromatography (HPLC) with UV or fluorescence detection are commonly used.

To the authors' best knowledge, determination of PAHs in PHA biopolymers has not been reported in the literature, yet. In the present work, a procedure to extract the sixteen priority PAHs from PHAs was optimized. The critical step was to select a solvent able to extract the target analytes without dissolving the PHA. Then, the extract was cleaned up by solid phase extraction (SPE), and PAHs were quantified by GC/MS with isotopic dilution method. The method was finally applied to two PHAs obtained by using the OFMSW as feedstock PHA $_{\mathrm{O}-}$ FMSW $)$ and a synthetic VFA mixture $\left(\mathrm{PHA}_{\text {synth }}\right)$.

\section{Experimental}

\subsection{Materials and reagents}

Sand $\left(\mathrm{SiO}_{2}\right.$; purum per analysis; acid purified; 40-200 mesh) was purchased from Sigma-Aldrich (Milan, Italy). Before use, the sand was washed with $1 \mathrm{~mol} \mathrm{~L}^{-1}$ hydrochloric acid, then with ultrapure water (five times) and finally with methanol (three times) to remove residual impurity.

Florisil $\left(\mathrm{MgO}_{3} \mathrm{Si}\right.$; $60-100$ mesh) was obtained from Carlo Erba (Milan, Italy). Florisil cartridges were prepared by filling $6 \mathrm{~mL}$ polypropylene tubes (Supelco, Bellefonte, PA, USA) with $500 \mathrm{mg}$ of the adsorbent placed between two polyethylene frits (Supelco).

Dichloromethane and $n$-hexane were of HPLC or GC grade and were purchased from Sigma-Aldrich (Milan, Italy), as well as hydrochloric acid. Acetonitrile of HPLC grade was purchased from VWR International (Milan, Italy). Ultrapure water (resistivity $18.2 \mathrm{M} \Omega \mathrm{cm}$ ) was obtained by an Arium 611 VF system from Sartorius (Göttingen, Germany).

\subsection{Standard solutions}

A standard mixture containing the US EPA sixteen priority PAHs (PAH calibration mix, TraceCERT ${ }^{\circ}$ grade, $10 \mu \mathrm{g} \mathrm{m}^{-1}$ each component in acetonitrile) was purchased from Supelco. The sixteen PAH mixture included naphthalene (NAP), acenaphthylene (ACY), acenaphthene (ACE), fluorene (FLU), phenanthrene (PHE), anthracene (ANT), fluoranthene (FLT), pyrene (PYR), benz[a]anthracene (BaA), chrysene $(\mathrm{CHR})$, benzo[b]fluoranthene $(\mathrm{BbF})$, benzo[k]fluoranthene $(\mathrm{BkF})$, benzo[a]pyrene (BaP), indeno[1,2,3-cd]pyrene (IPY), dibenz[a,h]anthracene (DBahA), and benzo[ghi]perylene (BPE). Structures are reported in the Supplementary material Fig. S1.

A mixture of fourteen perdeuterated PAHs (chemical purities $>$ $98 \%$, isotopic purities $\geq 98 \%$ ), concentration $100 \mu \mathrm{gL}^{-1}$ in isooctane/toluene 75:25 $(v / v)$, was obtained from Wellington laboratories (Guelph, ON, Canada). The deuterated internal standard (d-IS) mixture included naphthalene-d8 (NAP-d8), 2-methylnaphthalene-d10, acenaphthylene-d8 (ACY-d8), phenanthrene-d10 (PHE-d10), fluoranthened10 (FLT-d10), benz[a] anthracene-d12 (BaA-d12), chrysene-d12 (CHR$\mathrm{d} 12)$, benzo[b]fluoranthene-d12 (BbF-d12), benzo[k]fluoranthene-d12 (BkF-d12), benzo[a]pyrene-d12 (BaP-d12), perylene-d12 (PER-d12), indeno[1,2,3-c,d]pyrene-d12 (IPY-d12), dibenz[a,h]anthracene-d14 (DBahA-d14), and benzo[g,h,i]perylene-d12 (BPE-d12).
Recovery standard stock solution L429-RS, containing $100 \mathrm{ng} \mu \mathrm{L}^{-1}$ (1.2 mL in isooctane) of acenaphthene-d10 (ACE-d10), pyrene-d10 (PYR-d10) and benzo[e]pyrene-d12 (BeP-d12) was obtained from Wellington Laboratories.

\subsection{PHA samples}

The two PHA freeze-dried samples were kindly provided by Prof. Mauro Majone from the Chemistry Department of University of Rome "La Sapienza". Details on their production are reported in the Supplementary material. The PHA $\mathrm{OFMSw}_{\text {and }} \mathrm{PHA}_{\text {synth }}$ polymers were provided at $90 \%$ and $80 \%(w / w)$ purity, respectively.

\subsection{Instrumental analysis}

For preliminary experiments, a Shimadzu (Kyoto, Japan) HPLC system was used, which included two Nexera XR LC-20 AD dualplunger parallel flow pumps, a CBM-20A controller, a Prominence SPDM20A diode array detector, a DGU-20A3 online degasser, and a CTO$20 \mathrm{~A}$ column oven. The chromatographic column was a $250 \mathrm{~mm} \times 4.6 \mathrm{~mm}$ i.d. ( $5 \mu \mathrm{m}$ particle size) BEH C18 (Waters, Milford, MA, USA). Mobile phase was water/acetonitrile; the gradient profile, referring to acetonitrile percentage, was from $60 \%$ to $100 \%$ in $30 \mathrm{~min}$, followed by an equilibration step at $60 \%$ for $15 \mathrm{~min}$. Flow-rate was $0.5 \mathrm{~mL} \mathrm{~min}^{-1}$; injection volume was $10 \mu \mathrm{L}$. UV detection was carried out at 220 and $254 \mathrm{~nm}$ wavelengths.

$\mathrm{PAH}$ determination was carried out using a gas chromatograph coupled with a mass spectrometer, namely an ISQ $^{\mathrm{TM}}$ Series Single Quadrupole GC/MS (Thermo Fisher Scientific, St. Peters, MO, USA) equipped with a SLB ${ }^{\mathrm{TM}}-5 \mathrm{~ms}$ (Supelco, Milan, Italy) fused silica capillary GC column, poly $(5 \%$ diphenyl/95\% dimethyl siloxane) phase, $30 \mathrm{~m} \times 0.25 \mathrm{~mm}$ i.d., $0.25 \mu \mathrm{m}$ film thickness. He (99.9995\% purity) was used as carrier gas. The injector was a programmed-temperature vaporizing (PTV) inlet; the injection volume was $1 \mu \mathrm{L}$. Temperature programming was set as follows: the initial oven temperature was $40^{\circ} \mathrm{C}$, kept constant for $5 \mathrm{~min}$, then increased to $290^{\circ} \mathrm{C}$ with $12^{\circ} \mathrm{C} \min ^{-1}$ and kept constant for $6 \mathrm{~min}$; finally, the temperature was increased to $325^{\circ} \mathrm{C}$ with $20^{\circ} \mathrm{C} \mathrm{min}{ }^{-1}$ and kept constant for $15 \mathrm{~min}$. The temperature of transfer line was $270^{\circ} \mathrm{C}$. MS was operated in electron ionization mode (EI, $70 \mathrm{eV}$ ), and acquisition was performed using single ion monitoring (SIM) at the molecular ion of each PAH at the time windows corresponding to their retention times. Each $\mathrm{PAH}$ was quantified using its corresponding perdeuterated isotopologue or a structural omologue perdeuterated isotopologue as IS (see Supplementary material Table S2).

\subsection{Sample preparation}

\subsubsection{Sample extraction}

A $100 \mathrm{mg}$-PHA sample aliquot was cut into small pieces (ca. $2 \mathrm{~mm}$ large), dispersed with $800 \mathrm{mg}$ of washed sand in a ceramic mortar by blending with a pestle by the aid of liquid nitrogen. After about 10 min, a fine, dry and homogenous powder was obtained; then, the powder was transferred into a beaker. At this point, $3 \mathrm{~mL}$ acetonitrile was added to the powder to increase its wettability before spiking with the d-IS solution mix and, for recovery experiments, with the analyte calibration mix solution too. Solvents were allowed to evaporate for $2 \mathrm{~h}$ under the laboratory fume hood.

After that, the powder (ca. $900 \mathrm{mg}$ constituted by polymer and sand) was added with $10 \mathrm{~mL} n$-hexane and placed in an ultrasonic bath for $40 \mathrm{~min}$. After centrifugation at $2000 \mathrm{~g}$ for $20 \mathrm{~min}$ (to remove residual sand), the supernatant was collected; the procedure was repeated once. The supernatants (total volume $<20 \mathrm{~mL}$ ) were mixed and cleaned up by SPE.

\subsubsection{Sample clean-up}

Immediately prior to use, the SPE cartridge packed with florisil was 
first washed with $4 \mathrm{~mL}$ dichloromethane/ $n$-hexane $1: 1(v / v)$ and then conditioned with $4 \mathrm{~mL} n$-hexane.

The extract was loaded into the cartridge and allowed to pass by gravity. After washing the cartridge with $6 \mathrm{~mL} n$-hexane, analytes were eluted by $6 \mathrm{~mL}$ dichloromethane/n-hexane 1:1 $(v / v)$. The eluate was collected and solvent removed by evaporation first in a rotary evaporator and then under a gentle nitrogen stream up to ca. $20 \mu \mathrm{L}$. Finally, the residue was reconstituted with $n$-hexane to a final volume of $100 \mu \mathrm{L}$.

\subsubsection{Precautions}

Since PAHs are ubiquitous compounds, all the containers were extensively cleaned up and rinsed with $n$-hexane before use. As suggested in the literature $[14,15]$, all solutions were stored in amber glass to avoid analyte degradation by light, and during sample preparation plastic materials were avoided as much as possible, especially polyethylene, to prevent analyte loss by adsorption. Moreover, during sample preparation, foil-wrapped vials and glassware were used to protect PAHs from light.

Another critical point of the experimental procedure is the solvent removal from the extract, because the loss of more volatile compounds could occur [16] (see boiling points reported in Table S1). Therefore, all the evaporation steps were performed in a rotary evaporator and then under a gentle nitrogen stream at mild temperature conditions, avoiding the complete drying of the extract.

\subsection{Method performance}

Method performance parameters, such as trueness, precision (intraday and interday within- laboratory precision), linearity range, limit of detection (LOD) and limit of quantitation (LOQ) were assessed. For the evaluation of these parameters, only PHA $\mathrm{OFMsw}_{\text {was }}$ used.

The spiking procedure before extraction was the same previously described (see "Section 2.5.1. Sample extraction").

\subsubsection{Calibration graphs}

To construct the calibration graph, standard calibration solutions were prepared at six different concentrations levels by appropriate dilution of the $16 \mathrm{PAH}$ mix solution in $n$-hexane, and adding a constant amount of the d-IS solution (100 ng, corresponding to $1 \mathrm{mg} \mathrm{L}^{-1}$ ). The levels were in the range $0.001-10 \mathrm{mg} \mathrm{L}^{-1}$. Each solution was prepared in duplicate and injected twice, randomly; results were averaged to obtain a single calibration graph for each PAH. For all the PAHs, the ratio of the peak area of each analyte to that of the relative d-IS was plotted against the concentration of the corresponding analyte.

\subsubsection{Recovery}

Because blank matrix was not available, to evaluate the extraction and clean-up yield, samples were spiked before extraction with the d-IS mix at two concentration levels, namely 20 and $200 \mu \mathrm{gg}^{-1}$ (corresponding to a total amount of 2 and $20 \mathrm{ng}$, respectively). Then, before injection into the GC/MS system, $5 \mathrm{ng}$ of recovery standard was added [17]. See Supplementary material for detail.

\subsubsection{Trueness and precision}

Method trueness was evaluated by analyte apparent recovery $\left(R_{\mathrm{a}}\right.$, $\%)$ at two different spiking levels, these depending on the specific PAH blank value. $R_{a}$ was calculated by comparing the analyte to d-IS peak area ratio in PHA samples spiked before extraction with the standard calibration graph, after blank subtraction. See Supplementary material for detail.

Therefore, samples were spiked with the sixteen PAH calibration mix at 20,200 , and $400 \mu \mathrm{g} \mathrm{kg}^{-1}$, where 20 and $200 \mu \mathrm{gg}^{-1}$ levels were used only for the analytes not detected in the blank sample above LOQ, and 200 and $400 \mu \mathrm{gg}^{-1}$ levels were used for the other PAHs. For all concentration levels, five replicates were processed.

Precision of the method was assessed as within-laboratory precision, both intraday (repeatability) and interday (reproducibility), at the two concentration levels used for trueness estimation, by calculating the relative standard deviation (RSD). The intraday precision was expressed as RSDr of the trueness values of five spiked samples, for each concentration level, analyzed in the same day. The interday precision was expressed as $\mathrm{RSD}_{\mathrm{R}}$ of the recovery values of five spiked samples, for each concentration level, analyzed in five consecutive days.

\subsubsection{Limit of detection and quantitation}

Matrix-matched solutions were prepared at four concentration levels, namely $1,2,5$, and $10 \mathrm{ng} \mathrm{g}^{-1}\left(\mu \mathrm{kg}^{-1}\right)$ by spiking the PHA samples before extraction and following the entire analytical protocol.

For PAHs not naturally present in the PHA sample, method LODs were estimated as the lowest matrix-matched points giving at least a signal-to-noise ratio $(\mathrm{S} / \mathrm{N})>3$; method LOQs were estimated as the lowest matrix-matched points giving at least a $\mathrm{S} / \mathrm{N}>10$. For PAHs naturally present in the PHA sample (namely NAP, ACY, FLU, PHE, ANT, PYR, BaA, and CHR), method LODs and LOQs were estimated by preparing matrix-matched d-IS solutions at the same levels of the extrapolated LODs and LOQs for the other analytes.

\section{Results and discussion}

\subsection{Optimization of PAH extraction and clean-up procedure}

Method was developed using only the PHA OFMSw $_{\text {polymer. Different }}$ extraction solvents and techniques for $\mathrm{PAH}$ extraction were compared in terms of absolute recovery (see Supplementary material Table S3 and Fig. S2), by spiking the sample with $10 \mu \mathrm{L}$ PAH mixture (100 ng each $\mathrm{PAH})$. After solvent extraction, the clean-up step on florisil cartridge was needed to reduce matrix effect and improve sensitivity. At this first stage of method development, samples were analyzed by HPLC-UV (see Supplementary material for detail).

A first attempt to extract target analytes form PAH consisted in handling a $100 \mathrm{mg}$-aliquot of biopolymer into a separation funnel and adding $20 \mathrm{~mL}$ of a mixture dichloromethane/n-hexane $1: 1(v / v), 80 \mathrm{~mL}$ of ultrapure water, and ca. $300 \mathrm{mg} \mathrm{Na}_{2} \mathrm{SO}_{4}$. After $5 \mathrm{~min}$ manual shaking, the solution was allowed to separate for $5 \mathrm{~min}$ and then the upper layer, constituted by dichloromethane/ $n$-hexane, was collected and centrifuged to remove polymer and water residue. The upper layer was collected and after solvent removal into a rotary evaporator, the residue was reconstituted with $10 \mathrm{~mL} n$-hexane. Extract clean-up was performed by florisil SPE as described in experimental section. However, in this condition, a turbid solution was obtained and cartridge clogging occurred.

Even by reducing sample amount to $50 \mathrm{mg}$ and repeating the extraction with $80 \mathrm{~mL}$ of ultrapure water saturated with salt twice, unsatisfactory results were obtained. A further modification in the analytical procedure consisted in using dichloromethane/n-hexane 80:20 $(v / v)$ for PAH elution from the florisil SPE cartridge. Also in this case, unsatisfactory and irreproducible recovery values were obtained.

The last experiments were based on the previous dispersion of $100 \mathrm{mg}$ PHA with sand. In the optimized protocol, the resulting powder (polymer and sand) was added with $10 \mathrm{~mL} n$-hexane and placed in an ultrasonic bath for $40 \mathrm{~min}$. After centrifugation at $2000 \mathrm{~g}$ for $20 \mathrm{~min}$, the supernatant was collected; the procedure was repeated once and the supernatants were mixed and cleaned up by SPE. Beside $n$-hexane, also acetonitrile and acetonitrile $/ n$-hexane $1 / 1(v / v)$ were tested as extraction solvents.

\subsection{GC/MS analysis}

To achieve best performance and improve sensitivity [18], GC/MS with EI source and quadrupole mass analyzer was used for method validation. Indeed, when subject to EI, the condensed-ring PAH structures yield an intense molecular ion peak $\mathrm{M}^{+\cdot}$ with no or little 
fragmentation $[19,20]$. Isotope dilution technique by using fully deuterated analogues of target analytes was used for analyte quantitation. Indeed, GC/MS with isotope dilution should compensate analyte losses occurring during the various analytical procedure steps, making the results more accurate, precise, and selective [21].

\subsection{Method performance}

PHA blank samples were not available, as assessed by preliminary experiments with the optimized extraction procedure. In particular, 8/ 16 selected PAHs were detected in both polymers. For this reason, standard calibration graphs were used for quantitation. The tested linearity range $\left(0.001-10 \mathrm{mg} \mathrm{L}^{-1}\right)$ was appropriate to the levels found in the biopolymer samples, and with the limits set for certain plastic components by European Law [11]. The coefficients of determination ranged between 0.9930 and 1.0000 (see Supplementary material Table S4).

Because neither certified reference materials nor blank samples for PHAs were available, to assess method performance spiked samples were employed $[22,23]$. This method is commonly used in the literature, however, it cannot be assumed that the analyte is bound in the same way as it would be in a natural matrix [22]. To assure as much as possible a homogeneous distribution of the spiked analytes into the matrix, the solid biopolymer was first converted into a powder and then wetted with a large amount of acetonitrile before spiking the matrix with the analytes.

For each sample batch, a chromatographic control was performed by injecting at the beginning and at the end of the batch a standard solution containing all the sixteen PAHs, and an instrumental blank.

Extraction recovery was estimated using exclusively perdeuterated standards, as described in the Experimental section. The calculated recoveries reported in Table 1 show that more volatile and lower molecular weight PAHs, such as (deuterated) NAP, are particularly prone to losses during extraction and sample concentration, as already reported in the literature [13].

Trueness, within-laboratory intra-day (repeatability) and inter-day precision (intermediate precision) were estimated at two concentration levels within the linear range. Trueness was evaluated in terms of apparent recovery. For the determination of intra- and inter-day precisions five replicates for each level were analyzed on the same day and on five different days, respectively.

Analytical performance of the developed method is summarized in Supplementary material Table S5. Method LOQs varied between 5 and $10 \mu \mathrm{gg}^{-1}$, therefore they were suitable to assure biopolymer safety use.

\subsection{Application to samples}

After a previous check that method performance was not affected by PHA typology, analyses were performed on both PHA biopolymer

Table 1

Extraction recoveries (RSD; $n=4$ ) at two concentration levels.

\begin{tabular}{llll}
\hline $\begin{array}{l}\text { Deuterated } \\
\text { compound }\end{array}$ & $\begin{array}{l}\text { Recovery } \\
\text { standard }\end{array}$ & $\begin{array}{l}\text { Recovery (RSD; } \\
\text { \%) } 20 \mu \mathrm{kg}^{-1}\end{array}$ & $\begin{array}{l}\text { Recovery (RSD; \%) } \\
200 \mu \mathrm{kg}^{-1}\end{array}$ \\
\hline NAP-d8 & ACE-d10 & $56(16)$ & $63(19)$ \\
ACY-d8 & ACE-d10 & $59(10)$ & $68(14)$ \\
PHE-d10 & ACE-d10 & $89(7)$ & $92(5)$ \\
FLT-d10 & PYR-d10 & $96(2)$ & $92(7)$ \\
BaA-d12 & PYR-d10 & $94(4)$ & $97(8)$ \\
CHR-d12 & PYR-d10 & $93(11)$ & $95(6)$ \\
BbF-d12 & BeP-d12 & $99(12)$ & $95(2)$ \\
BkF-d12 & BeP-d12 & $100(4)$ & $99(2)$ \\
BaP-d12 & BeP-d12 & $97(6)$ & $94(11)$ \\
IPY-d12 & BeP-d12 & $95(8)$ & $96(5)$ \\
DBahA-d14 & BeP-d12 & $98(1)$ & $99(4)$ \\
BPE-d12 & BeP-d12 & $96(3)$ & $101(3)$ \\
\hline
\end{tabular}

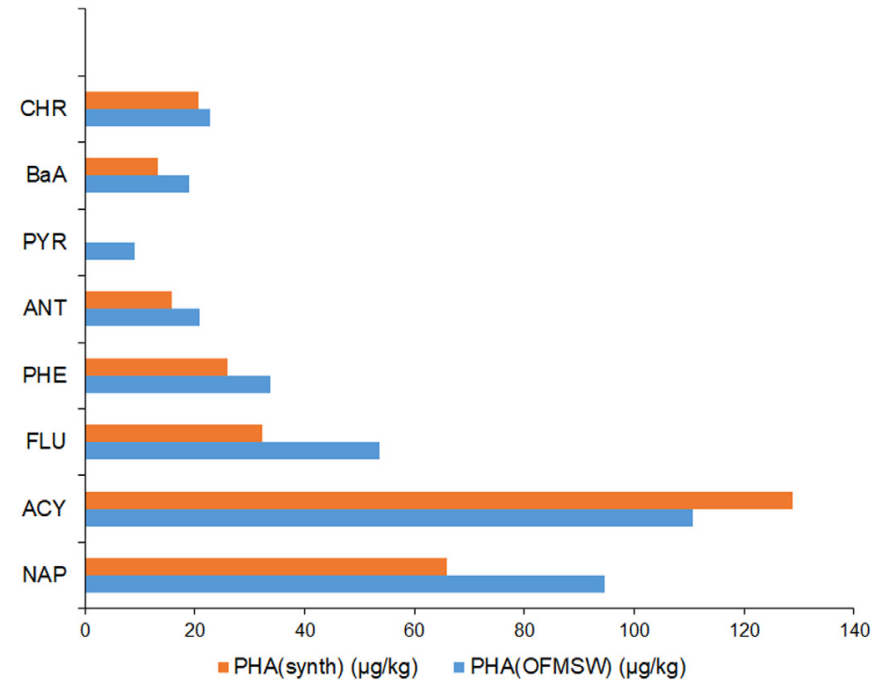

Fig. 1. Results obtained by the analysis of the two PHA sample typologies.

samples. Results are reported in Fig. 1 (see also Supplementary material Table S6). Results indicated that the $\mathrm{PHA}_{\mathrm{OFMSW}}$ and $\mathrm{PHA}_{\text {synth }}$ were contaminated by the same PAHs (eight and seven PAHs, respectively) at comparable levels. The $\mathrm{PHA}_{\text {synth }}$ was less contaminated than PHA $\mathrm{OFMSW}_{\mathrm{O}}$ and this was very likely due to the different origin.

\section{Conclusion}

Among bioproducts, the PHA biopolymers are attracting increasing attention for their versatility, although their high production cost limits their diffusion. Therefore, the possibility to obtain them from organic waste material is an interesting prospective from the economical point of view. However, to do this, it is necessary to have at disposable rapid, multiresidual and sensitive analytical methods assessing the absence of toxic compounds potentially present in the feedstock used for their production.

For this reason, the present work, in the context of the European circular economy strategy, was aimed to develop an analytical method for the determination of PAHs at trace levels in PHA biopolymers. The sixteen selected PAHs, representing the US EPA priority compounds, were analyzed by GC/MS analysis after solvent extraction and following clean-up on a classical normal phase adsorbent. The obtained LOQs resulted suitable for determining the analytes well below the limits set for some plastic components of articles of general use set by European regulation. Two different PHAs were analyzed, one produced by organic municipal waste and another one via synthetic pathway. The detected PAHs were the same and at comparable levels in both PHAs, meaning that the feedstock source has a minimal impact on the final product contamination.

\section{Acknowledgements}

We would like to thank Prof. Mauro Majone from Department of Chemistry of University of Rome "La Sapienza" for providing us the PHA samples.

\section{Funding}

This work was supported by the REsources from URban BIo-waSte" - RES URBIS (GA 7303499) project in the European Horizon2020 (Call CIRC-05-2016) program.

The funding source did not have any role in study design, in the collection, analysis and interpretation of data, in the writing of the report, and in the decision to submit the article for publication. 


\section{Conflict of interest}

None.

\section{Appendix A. Supplementary material}

Supplementary data associated with this article can be found in the online version at http://dx.doi.org/10.1016/j.talanta.2018.06.038.

\section{References}

[1] R. Khiewwijit, K.J. Keesman, H. Rijnaarts, H. Temmink, Volatile fatty acids production from sewage organic matter by combined bioflocculation and anaerobic fermentation, Bioresour. Technol. 193 (2015) 150-155, http://dx.doi.org/10 1016/j.biortech.2015.06.103.

[2] F. Valentino, F. Morgan-Sagastume, S. Campanari, M. Villano, A. Werker, M. Majone, Carbon recovery from wastewater through bioconversion into biodegradable polymers, New Biotechnol. 37 (2017) 9-23, http://dx.doi.org/10.1016/j. nbt.2016.05.007.

[3] M.R. Kosseva, E. Rusbandi, Trends in the biomanufacture of polyhydroxyalkanoates with focus on downstream processing, Int. J. Biol. Macromol. (2017), http://dx.doi. org /10.1016/j.ijbiomac.2017.09.054.

[4] L.I. Blake, F.A. Halim, C. Gray, R. Mair, D.A.C. Manning, P. Sallis, H. Hutchinson, N.D. Gray, Evaluating an anaerobic digestion (AD) feedstock derived from a novel non-source segregated municipal solid waste (MSW) product, Waste Manag. 59 (2017) 149-159, http://dx.doi.org/10.1016/j.wasman.2016.10.031.

[5] R.C. Brändli, T.D. Bucheli, T. Kupper, J. Mayer, F.X. Stadelmann, J. Tarradellas, Fate of PCBs, PAHs and their source characteristic ratios during composting and digestion of source-separated organic waste in full-scale plants, Environ. Pollut. 148 (2007) 520-528, http://dx.doi.org/10.1016/j.envpol.2006.11.021.

[6] R.C. Brändli, T.D. Bucheli, T. Kupper, R. Furrer, F.X. Stadelmann, J. Tarradellas, Persistent organic pollutants in source-separated compost and its feedstock materials-a review of field studies, J. Environ. Qual. 34 (2005) 735, http://dx.doi.org/ 10.2134/jeq.2004.0333.

[7] I. Siebielska, Comparison of changes in selected polycyclic aromatic hydrocarbons concentrations during the composting and anaerobic digestion processes of municipal waste and sewage sludge mixtures, Water Sci. Technol. 70 (2014) 1617-1624, http://dx.doi.org/10.2166/wst.2014.417.

[8] K.-H. Kim, S.A. Jahan, E. Kabir, R.J.C. Brown, A review of airborne polycyclic aromatic hydrocarbons (PAHs) and their human health effects, Environ. Int. 60 (2013) 71-80, http://dx.doi.org/10.1016/j.envint.2013.07.019.

[9] International Agency for Research on Cancer (IARC), IARC Monographs on the Evaluation of Carcinogenic Risks to Humans, (n.d.) volumes 1-120. 〈http://
monographs.iarc.fr/ENG/Classification/latest_classif.php> (Accessed 6 December 2017).

[10] Directive 2004/107/EC of the European Parliament and of the Council of 15/12/ 2004 relating to arsenic, cadmium, mercury, nickel and polycyclic aromatic hydrocarbons in ambient air, Off. J. Eur. Union, 23, 2005, pp. 3-16.

[11] Regulation (EC) 1907/2006 of the European Parliament and of the Council of 18 December 2006 - REACH, Off. J. Eur. Union, 2006, pp. 396-849.

[12] Regulation (EC) No $1881 / 2006$ of 19 December 2006 setting maximum levels for certain contaminants in foodstuffs, Off. J. Eur. Union, L364, 2006, pp. 5-24.

[13] J.E. Szulejko, K.H. Kim, R.J.C. Brown, M.S. Bae, Review of progress in solventextraction techniques for the determination of polyaromatic hydrocarbons as airborne pollutants, TrAC - Trends Anal. Chem. 61 (2014) 40-48, http://dx.doi.org/ 10.1016/j.trac.2014.07.001.

[14] G. Purcaro, S. Moret, L.S. Conte, Overview on polycyclic aromatic hydrocarbons: occurrence, legislation and innovative determination in foods, Talanta 105 (2013) 292-305, http://dx.doi.org/10.1016/j.talanta.2012.10.041.

[15] P. Plaza-Bolaños, A.G. Frenich, J.L.M. Vidal, Polycyclic aromatic hydrocarbons in food and beverages. Analytical methods and trends, J. Chromatogr. A 1217 (2010) 6303-6326, http://dx.doi.org/10.1016/j.chroma.2010.07.079.

[16] C.C. Cheng, Recovery of polycyclic aromatic hydrocarbons during solvent evaporation with a rotary evaporator, Polycycl. Aromat. Compd. 23 (2003) 315-325, http://dx.doi.org/10.1080/10406630308048.

[17] V. Paolini, E. Guerriero, A. Bacaloni, M. Rotatori, P. Benedetti, S. Mosca, Simultaneous sampling of vapor and particle-phase carcinogenic polycyclic aromatic hydrocarbons on functionalized glass fiber filters, Aerosol Air Qual. Res. 16 (2016) 175-183, http://dx.doi.org/10.4209/aaqr.2015.07.0476.

[18] D.L. Poster, M.M. Schantz, L.C. Sander, S.A. Wise, Analysis of polycyclic aromatic hydrocarbons (PAHs) in environmental samples: a critical review of gas chromatographic (GC) methods, Anal. Bioanal. Chem. 386 (2006) 859-881, http://dx.doi. org/10.1007/s00216-006-0771-0.

[19] A. Paris, J. Ledauphin, P. Poinot, J.-L. Gaillard, Polycyclic aromatic hydrocarbons in fruits and vegetables: origin, analysis, and occurrence, Environ. Pollut. 234 (2018) 96-106, http://dx.doi.org/10.1016/j.envpol.2017.11.028.

[20] J. De Boer, R.J. Law, Developments in the use of chromatographic techniques in marine laboratories for the determination of halogenated contaminants and polycyclic aromatic hydrocarbons, J. Chromatogr. A 1000 (2003) 223-251, http://dx. doi.org/10.1016/S0021-9673(03)00309-1.

[21] S. Kumar, S. Negi, P. Maiti, Biological and analytical techniques used for detection of polyaromatic hydrocarbons, Environ. Sci. Pollut. Res. (2017), http://dx.doi.org/ 10.1007/s11356-017-0415-2.

[22] AOAC International, Guidelines for standard method performance requirements (Appendix F), AOAC Off. Methods Anal. (2016) 1-18.

[23] M. Thompson, S.L.R. Ellison, R. Wood, Harmonized guidelines for single-laboratory validation of methods of analysis (IUPAC Technical Report), Pure Appl. Chem. 74 (2002), http://dx.doi.org/10.1351/pac200274050835. 